\title{
An inverse problem for a quasilinear parabolic equation with nonlocal boundary and overdetermination conditions
}

Fatma Kanca ${ }^{\text {* }}$ and Irem Baglan²

\section{"Correspondence:}

fatma.kanca@khas.edu.tr

'Department of Management Information Systems, Kadir Has

University, Istanbul, 34083, Turkey

Full list of author information is

available at the end of the article

\begin{abstract}
In this paper the inverse problem of finding the time-dependent coefficient of heat capacity together with the solution of heat equation with nonlocal boundary conditions is considered. Under some natural regularity and consistency conditions on the input data, the existence, uniqueness and continuous dependence upon the data of the solution are shown. Some considerations on the numerical solution for this inverse problem are presented with an example.
\end{abstract}

\section{Introduction}

Denote the domain $D$ by

$$
D:=\{0<x<1,0<t<T\} .
$$

Consider the equation

$$
u_{t}=u_{x x}-p(t) u+f(x, t, u)
$$

with the initial condition

$$
u(x, 0)=\varphi(x), \quad x \in[0,1]
$$

the nonlocal boundary condition

$$
u(0, t)=0, \quad u_{x}(0, t)=u_{x}(1, t), \quad t \in[0, T],
$$

and the integral overdetermination data

$$
\int_{0}^{1} u(x, t) d x=E(t), \quad 0 \leq t \leq T
$$

for a quasilinear parabolic equation with the nonlinear source term $f=f(x, t, u)$.

The functions $\varphi(x)$ and $f(x, t, u)$ are given functions on $[0,1]$ and $\bar{D} \times(-\infty, \infty)$, respectively.

The problem of finding the pair $\{p(t), u(x, t)\}$ in (1)-(4) will be called an inverse problem.

2014 Kanca and Baglan; licensee Springer. This is an Open Access article distributed under the terms of the Creative Commons Attribution License (http://creativecommons.org/licenses/by/2.0), which permits unrestricted use, distribution, and reproduction in any medium, provided the original work is properly cited. 
Definition 1 The pair $\{p(t), u(x, t)\}$ from the class $C[0, T] \times\left(C^{2,1}(D) \cap C^{1,0}(\bar{D})\right)$, for which conditions (1)-(4) are satisfied and $p(t) \geq 0$ on the interval $[0, T]$, is called the classical solution of inverse problem (1)-(4).

The problem of identifying a coefficient in a nonlinear parabolic equation is an interesting problem for many scientists [1-3].

Inverse problems for parabolic equations with nonlocal boundary conditions are investigated in $[4,5]$. This kind of conditions arise from many important applications in heat transfer, life sciences, etc. In [6], also the nature of (3) type boundary conditions is demonstrated.

These kind of conditions such as (4) arise from many important applications in heat transfer, thermoelasticity, control theory, life sciences, etc. For example, in heat propagation in a thin rod, in which the law of variation $E(t)$ of the total quantity of heat in the rod is given in [7].

The paper organized as follows.

In Section 2, the existence and uniqueness of the solution of inverse problem (1)-(4) are proved by using the Fourier method and the iteration method. In Section 3, continuous dependence upon the data of the inverse problem is shown. In Section 4, the numerical procedure for the solution of the inverse problem is given.

\section{Existence and uniqueness of the solution of the inverse problem}

Consider the following system of functions on the interval $[0,1]$ :

$$
\begin{array}{ll}
X_{0}(x)=x, & X_{2 k-1}(x)=x \cos (2 \pi k x), \quad X_{2 k}(x)=\sin (2 \pi k x), \quad k=1,2, \ldots, \\
Y_{0}(x)=2, \quad Y_{2 k-1}(x)=4 \cos (2 \pi k x), & Y_{2 k}(x)=4(1-x) \sin (2 \pi k x), \quad k=1,2, \ldots
\end{array}
$$

The systems of these functions arise in [8] for the solution of a nonlocal boundary value problem in heat conduction. It is easy to verify that the systems of functions $X_{k}(x)$ and $Y_{k}(x), k=0,1,2, \ldots$, are biorthonormal on $[0,1]$. They are also Riesz bases in $L_{2}[0,1]$ (see $[5,9])$.

The main result on the existence and uniqueness of the solution of inverse problem (1)(4) is presented as follows.

We have the following assumptions on the data of problem (1)-(4):

$\left(\mathrm{A}_{1}\right) \quad E(t) \in C^{1}[0, T], E(t)>0, E^{\prime}(t) \leq 0$.

$\left(\mathrm{A}_{2}\right)\left(\mathrm{A}_{2}\right)_{1} \varphi(x) \in C^{3}[0,1]$,

$\left(\mathrm{A}_{2}\right)_{2} \varphi(0)=0, \varphi^{\prime}(0)=\varphi^{\prime}(1), \varphi^{\prime \prime}(0)=0$.

$\left(\mathrm{A}_{3}\right)\left(\mathrm{A}_{3}\right)_{1}$ Let the function $f(x, t, u)$ be continuous with respect to all arguments in $\bar{D} \times$ $(-\infty, \infty)$ and satisfy the following condition:

$$
\left|\frac{\partial^{(n)} f(x, t, u)}{\partial x^{n}}-\frac{\partial^{(n)} f(x, t, \tilde{u})}{\partial x^{n}}\right| \leq b(t, x)|u-\tilde{u}|, \quad n=0,1,2,
$$

where $b(x, t) \in L_{2}(D), b(x, t) \geq 0$.

$\left(\mathrm{A}_{3}\right)_{2} f(x, t, u) \in C^{3}[0,1], t \in[0, T]$,

$\left.\left(\mathrm{A}_{3}\right)_{3} f(x, t, u)\right|_{x=0}=0,\left.f_{x}(x, t, u)\right|_{x=0}=\left.f_{x}(x, t, u)\right|_{x=1},\left.f_{x x}(x, t, u)\right|_{x=0}=0$,

$\left(\mathrm{A}_{3}\right)_{4} f_{0}(t) \geq 0, \forall t \in[0, T]$ 
where

$$
\varphi_{k}=\int_{0}^{1} \varphi(x) Y_{k}(x) d x, \quad f_{k}(t)=\int_{0}^{1} f(x, t, u) Y_{k}(x) d x, \quad k=0,1,2, \ldots
$$

By applying the standard procedure of the Fourier method, we obtain the following representation for the solution of (1)-(3) for arbitrary $p(t) \in C[0, T]$ :

$$
\begin{aligned}
& u(x, t)=\left[\varphi_{0} e^{-\int_{0}^{t} p(s) d s}+2 \int_{0}^{t} \int_{0}^{1} f(\xi, \tau, u) e^{-\int_{\tau}^{t} p(s) d s} d \xi d \tau\right] X_{0}(x) \\
& +\sum_{k=1}^{\infty} X_{2 k-1}(x)\left[\varphi_{2 k-1} e^{-(2 \pi k)^{2} t-\int_{0}^{t} p(s) d s}\right. \\
& \left.+4 \int_{0}^{t} \int_{0}^{1} f(\xi, \tau, u) \cos 2 \pi k \xi e^{-(2 \pi k)^{2}(t-\tau)-\int_{\tau}^{t} p(s) d s} d \xi d \tau\right] \\
& +\sum_{k=1}^{\infty} X_{2 k}(x)\left[\left(\varphi_{2 k}-4 \pi k t \varphi_{2 k-1}\right) e^{-(2 \pi k)^{2} t-\int_{0}^{t} p(s) d s}\right] \\
& +\sum_{k=1}^{\infty} X_{2 k}(x)\left[4 \int_{0}^{t} \int_{0}^{1} f(\xi, \tau, u)(1-\xi) \sin 2 k \xi e^{-(2 \pi k)^{2}(t-\tau)-\int_{\tau}^{t} p(s) d s} d \xi d \tau\right] \\
& -\sum_{k=1}^{\infty} 4 \pi k X_{2 k}(x) \\
& \times\left[4 \int_{0}^{t} \int_{0}^{1} f(\xi, \tau, u)(t-\tau) \cos 2 \pi k \xi e^{-(2 \pi k)^{2}(t-\tau)-\int_{\tau}^{t} p(s) d s} d \xi d \tau\right], \\
& u_{0}(t)=\varphi_{0} e^{-\int_{0}^{t} p(s) d s}+\int_{0}^{t} \int_{0}^{1} f(\xi, \tau, u) \xi e^{-\int_{\tau}^{t} p(s) d s} d \xi d \tau, \\
& u_{2 k}(t)=\left[\left(\varphi_{2 k}-4 \pi k t \varphi_{2 k-1}\right) e^{-(2 \pi k)^{2} t-\int_{0}^{t} p(s) d s}\right] \\
& +\left[4 \int_{0}^{t} \int_{0}^{1} f(\xi, \tau, u)(1-\xi) \sin 2 \pi k \xi e^{-(2 \pi k)^{2}(t-\tau)-\int_{\tau}^{t} p(s) d s} d \xi d \tau\right] \\
& -4 \pi k\left[4 \int_{0}^{t} \int_{0}^{1} f(\xi, \tau, u)(t-\tau) \cos 2 \pi k \xi e^{-(2 \pi k)^{2}(t-\tau)-\int_{\tau}^{t} p(s) d s} d \xi d \tau\right] \text {, } \\
& u_{2 k-1}(t)=\left[\varphi_{2 k-1} e^{-(2 \pi k)^{2} t-\int_{0}^{t} p(s) d s}\right. \\
& \left.+4 \int_{0}^{t} \int_{0}^{1} f(\xi, \tau, u) \cos 2 \pi k \xi e^{-(2 \pi k)^{2}(t-\tau)-\int_{\tau}^{t} p(s) d s} d \xi d \tau\right] .
\end{aligned}
$$

Under conditions $\left(\mathrm{A}_{2}\right)_{1}$ and $\left(\mathrm{A}_{3}\right)_{2}$, the series (5) and $\sum_{k=1}^{\infty} \frac{\partial}{\partial x}$ converge uniformly in $\bar{D}$ since their majorizing sums are absolutely convergent. Therefore their sums $u(x, t)$ and $u_{x}(x, t)$ are continuous in $\bar{D}$. In addition, the series $\sum_{k=1}^{\infty} \frac{\partial}{\partial t}$ and $\sum_{k=1}^{\infty} \frac{\partial^{2}}{\partial x^{2}}$ are uniformly convergent for $t \geq \varepsilon>0$ ( $\varepsilon$ is an arbitrary positive number). In addition, $u_{t}(x, t)$ is continuous in $\bar{D}$ because the majorizing sum of $\sum_{k=1}^{\infty} \frac{\partial}{\partial t}$ is absolutely convergent under conditions $\left(\mathrm{A}_{2}\right)_{2}$ and $\left(\mathrm{A}_{3}\right)_{3}$. Differentiating $(4)$ under condition $\left(\mathrm{A}_{1}\right)$, we obtain

$$
\int_{0}^{1} u_{t}(x, t) d x=E^{\prime}(t), \quad 0 \leq t \leq T
$$


Equations (5) and (6) yield

$$
p(t)=\frac{1}{E(t)}\left[-E^{\prime}(t)+\frac{1}{2} f_{0}(t)\right]
$$

Definition 2 Denote the set

$$
\{u(t)\}=\left\{u_{0}(t), u_{2 k}(t), u_{2 k-1}(t), k=1, \ldots, n\right\}
$$

of continuous on $[0, T]$ functions satisfying the condition

$$
\max _{0 \leq t \leq T}\left|u_{0}(t)\right|+\sum_{k=1}^{\infty}\left(\max _{0 \leq t \leq T}\left|u_{2 k}(t)\right|+\max _{0 \leq t \leq T}\left|u_{2 k-1}(t)\right|\right)<\infty
$$

by $\mathbf{B}_{1}$. Let

$$
\|u(t)\|=\max _{0 \leq t \leq T}\left|u_{0}(t)\right|+\sum_{k=1}^{\infty}\left(\max _{0 \leq t \leq T}\left|u_{2 k}(t)\right|+\max _{0 \leq t \leq T}\left|u_{2 k-1}(t)\right|\right)
$$

be the norm in $\mathbf{B}_{1}$.

Let us denote

$$
\mathbf{B}_{2}=\{p(t) \in C[0, T]: p(t) \geq 0\},
$$

$\|p(t)\|=\max _{0 \leq t \leq T}|p(t)|$ be the norm in $\mathbf{B}_{2}$.

It can be shown that $\mathbf{B}_{1}$ and $\mathbf{B}_{2}$ are the Banach spaces.

Theorem 3 Let assumptions ( $\left.\mathrm{A}_{1}\right)-\left(\mathrm{A}_{3}\right)$ be satisfied. Then inverse problem (1)-(4) has a unique solution.

Proof An iteration for (5) is defined as follows:

$$
\begin{aligned}
u_{0}^{(N+1)}(t)= & u_{0}^{(0)}(t)+2 \int_{0}^{t} \int_{0}^{1} f\left(\xi, \tau, u^{(N)}\right) e^{-\int_{\tau}^{t} p^{(N)}(s) d s} d \xi d \tau, \\
u_{2 k-1}^{(N+1)}(t)= & u_{2 k-1}^{(0)}(t)+4 \int_{0}^{t} \int_{0}^{1} f\left(\xi, \tau, u^{(N)}\right) \cos 2 \pi k \xi e^{-(2 \pi k)^{2}(t-\tau)-\int_{\tau}^{t} p^{(N)}(s) d s} d \xi d \tau, \\
u_{2 k}^{(N+1)}(t)= & u_{2 k}^{(0)}(t)+4 \int_{0}^{t} \int_{0}^{1} f\left(\xi, \tau, u^{(N)}\right)(1-\xi) \sin 2 \pi k \xi e^{-(2 \pi k)^{2}(t-\tau)-\int_{\tau}^{t} p^{(N)}(s) d s} d \xi d \tau \\
& -16 \pi k \int_{0}^{t} \int_{0}^{1} f\left(\xi, \tau, u^{(N)}\right)(t-\tau) \cos 2 \pi k \xi e^{-(2 \pi k)^{2}(t-\tau)-\int_{\tau}^{t} p^{(N)}(s) d s} d \xi d \tau,
\end{aligned}
$$

where $N=0,1,2, \ldots$ and

$$
\begin{aligned}
& u_{0}^{(0)}(t)=\varphi_{0} e^{-\int_{0}^{t} p(s) d s}, \quad u_{2 k}^{(0)}(t)=\left(\varphi_{2 k}-4 \pi k t \varphi_{2 k-1}\right) e^{-(2 \pi k)^{2} t-\int_{0}^{t} p(s) d s}, \\
& u_{2 k-1}^{(0)}(t)=\varphi_{2 k-1} e^{-(2 \pi k)^{2} t-\int_{0}^{t} p(s) d s} .
\end{aligned}
$$

From the conditions of the theorem, we have $u^{(0)}(t) \in \mathbf{B}_{1}$ and $p^{(0)} \in \mathbf{B}_{2}$. 
Let us write $N=0$ in (8).

$$
u_{0}^{(1)}(t)=u_{0}^{(0)}(t)+2 \int_{0}^{t} \int_{0}^{1} f\left(\xi, \tau, u^{(0)}\right) d \xi d \tau
$$

Adding and subtracting $2 \int_{0}^{t} \int_{0}^{1} f(\xi, \tau, 0) d \xi d \tau$ to and from both sides of the last equation, we obtain

$$
u_{0}^{(1)}(t)=u_{0}^{(0)}(t)+2 \int_{0}^{t} \int_{0}^{1}\left[f\left(\xi, \tau, u^{(0)}(\xi, \tau)\right)-f(\xi, \tau, 0)\right] d \xi d \tau+2 \int_{0}^{t} \int_{0}^{1} f(\xi, \tau, 0) d \xi d \tau
$$

Applying the Cauchy inequality and the Lipschitz condition to the last equation and taking the maximum of both sides of the last inequality yields the following:

$$
\begin{gathered}
\max _{0 \leq t \leq T}\left|u_{0}^{(1)}(t)\right| \leq\left|\varphi_{0}\right|+\sqrt{T}\|b(x, t)\|_{L_{2}(D)}\left\|u^{(0)}(t)\right\|_{\mathbf{B}_{1}}+\sqrt{T}\|f(x, t, 0)\|_{L_{2}(D)}, \\
u_{2 k-1}^{(1)}(t)=\varphi_{2 k-1} e^{-(2 \pi k)^{2} t}+4 \int_{0}^{t} \int_{0}^{1}\left[f\left(\xi, \tau, u^{(0)}\right)-f(\xi, \tau, 0)\right] \cos 2 \pi k \xi e^{-(2 \pi k)^{2}(t-\tau)} d \xi d \tau \\
+4 \int_{0}^{t} \int_{0}^{1} f(\xi, \tau, 0) \cos 2 \pi k \xi e^{-(2 \pi k)^{2}(t-\tau)} d \xi d \tau .
\end{gathered}
$$

Applying the Cauchy inequality, the Hölder inequality, the Bessel inequality, the Lipschitz condition and taking maximum of both sides of the last inequality yields the following:

$$
\sum_{k=1}^{\infty} \max _{0 \leq t \leq T}\left|u_{2 k-1}^{(1)}(t)\right| \leq \sum_{k=1}^{\infty}\left|\varphi_{2 k-1}\right|+\frac{\sqrt{3}}{3}\|b(x, t)\|_{L_{2}(D)}\left\|u^{(0)}(t)\right\|_{\mathbf{B}_{1}}+\frac{\sqrt{3}}{3}\|f(x, t, 0)\|_{L_{2}(D)} .
$$

Applying the same estimations, we obtain

$$
\begin{aligned}
& \sum_{k=1}^{\infty} \max _{0 \leq t \leq T}\left|u_{2 k}^{(1)}(t)\right| \\
& \leq \sum_{k=1}^{\infty}\left|\varphi_{2 k}\right|+\frac{\sqrt{6} T}{3} \sum_{k=1}^{\infty}\left|\varphi_{2 k-1}^{\prime \prime}\right| \\
& \quad+\left(\frac{\sqrt{3}}{3}+2 \sqrt{2}|T|\right)\|b(x, t)\|_{L_{2}(D)}\left\|u^{(0)}(t)\right\|_{B_{1}}+\left(\frac{\sqrt{3}}{3}+2 \sqrt{2}|T|\right)\|f(x, t, 0)\|_{L_{2}(D)} .
\end{aligned}
$$

Finally, we have the following inequality:

$$
\begin{aligned}
\left\|u^{(1)}(t)\right\|_{\mathbf{B}_{1}}= & \max _{0 \leq t \leq T}\left|u_{0}^{(1)}(t)\right|+\sum_{k=1}^{\infty}\left(\max _{0 \leq t \leq T}\left|u_{2 k}^{(1)}(t)\right|+\max _{0 \leq t \leq T}\left|u_{2 k-1}^{(1)}(t)\right|\right) \\
\leq & \left|\varphi_{0}\right|+\sum_{k=1}^{\infty}\left(\left|\varphi_{2 k}\right|+\left|\varphi_{2 k-1}\right|\right)+\frac{\sqrt{6} T}{3} \sum_{k=1}^{\infty}\left|\varphi_{2 k-1}^{\prime \prime}\right| \\
& +\left(2 \sqrt{T}+\frac{2 \sqrt{3}}{3}+4 \sqrt{2}|T|\right)\|b(x, t)\|_{L_{2}(D)}\left\|u^{(0)}(t)\right\|_{\mathbf{B}_{1}} \\
& +\left(2 \sqrt{T}+\frac{2 \sqrt{3}}{3}+4 \sqrt{2}|T|\right)\|f(x, t, 0)\|_{L_{2}(D)} .
\end{aligned}
$$


Hence $u^{(1)}(t) \in \mathbf{B}_{1}$. In the same way, for a general value of $N$, we have

$$
\begin{aligned}
\left\|u^{(N)}(t)\right\|_{\mathbf{B}_{1}}= & \max _{0 \leq t \leq T}\left|u_{0}^{(N)}(t)\right|+\sum_{k=1}^{\infty}\left(\max _{0 \leq t \leq T}\left|u_{2 k}^{(N)}(t)\right|+\max _{0 \leq t \leq T}\left|u_{2 k-1}^{(N)}(t)\right|\right) \\
\leq & \left|\varphi_{0}\right|+\sum_{k=1}^{\infty}\left(\left|\varphi_{2 k}\right|+\left|\varphi_{2 k-1}\right|\right)+\frac{\sqrt{6} T}{3} \sum_{k=1}^{\infty}\left|\varphi_{2 k-1}^{\prime \prime}\right| \\
& +\left(2 \sqrt{T}+\frac{2 \sqrt{3}}{3}+4 \sqrt{2}|T|\right)\|b(x, t)\|_{L_{2}(D)}\left\|u^{(N-1)}(t)\right\|_{\mathbf{B}_{1}} \\
& +\left(2 \sqrt{T}+\frac{2 \sqrt{3}}{3}+4 \sqrt{2}|T|\right)\|f(x, t, 0)\|_{L_{2}(D)} .
\end{aligned}
$$

Since $u^{(N-1)}(t) \in \mathbf{B}_{1}$, we have $u^{(N)}(t) \in \mathbf{B}_{1}$

$$
\{u(t)\}=\left\{u_{0}(t), u_{2 k}(t), u_{2 k-1}(t), k=1,2, \ldots\right\} \in \mathbf{B}_{1} .
$$

An iteration for (7) is defined as follows:

$$
p^{(N)}(t)=\frac{1}{E(t)}\left[-E^{\prime}(t)+\frac{1}{2} \int_{0}^{1} f\left(\xi, \tau, u^{(N)}\right) d \xi\right]
$$

where $N=0,1,2, \ldots$

$$
p^{(1)}(t)=\frac{1}{E(t)}\left[-E^{\prime}(t)+\frac{1}{2} \int_{0}^{1} f\left(\xi, \tau, u^{(1)}\right) d \xi\right]
$$

Applying the Cauchy inequality,

$$
\left\|p^{(1)}(t)\right\|_{\mathbf{B}_{2}} \leq\left|\frac{-E^{\prime}(t)}{E(t)}\right|+\frac{1}{E(t)}\|b(x, t)\|_{L_{2}(D)}\left\|u^{(1)}(t)\right\|_{\mathbf{B}_{1}}+\frac{1}{E(t)}|f(x, t, 0)| .
$$

Hence $p^{(1)}(t) \in \mathbf{B}_{2}$. In the same way, for a general value of $N$, we have

$$
\left\|p^{(N)}(t)\right\|_{\mathbf{B}_{2}} \leq\left|\frac{-E^{\prime}(t)}{E(t)}\right|+\frac{1}{E(t)}\|b(x, t)\|_{L_{2}(D)}\left\|u^{(N)}(t)\right\|_{\mathbf{B}_{1}}+\frac{1}{E(t)}|f(x, t, 0)|,
$$

we deduce that $p^{(N)}(t) \in \mathbf{B}_{2}$.

Now we prove that the iterations $u^{(N+1)}(t)$ and $p^{(N+1)}(t)$ converge as $N \rightarrow \infty$ in $\mathbf{B}_{1}$ and $\mathbf{B}_{2}$, respectively.

$$
\begin{aligned}
& u_{0}^{(1)}(t)-u_{0}^{(0)}(t) \\
& =2 \int_{0}^{t} \int_{0}^{1}\left[f\left(\xi, \tau, u^{(0)}(\xi, \tau)\right)-f(\xi, \tau, 0)\right] d \xi d \tau+2 \int_{0}^{t} \int_{0}^{1} f(\xi, \tau, 0) d \xi d \tau, \\
& u_{2 k-1}^{(1)}(t)-u_{2 k-1}^{(0)}(t) \\
& =4 \int_{0}^{t} \int_{0}^{1}\left[f\left(\xi, \tau, u^{(0)}(\xi, \tau)\right)-f(\xi, \tau, 0)\right] e^{-(2 \pi k)^{2}(t-\tau)} \cos 2 \pi k \xi d \xi d \tau \\
& \quad+4 \int_{0}^{t} \int_{0}^{1} f(\xi, \tau, 0) e^{-(2 \pi k)^{2}(t-\tau)} \cos 2 \pi k \xi d \xi d \tau,
\end{aligned}
$$




$$
\begin{aligned}
u_{2 k}^{(1)}(t) & -u_{2 k}^{(0)}(t) \\
= & 4 \int_{0}^{t} \int_{0}^{1}\left[f\left(\xi, \tau, u^{(0)}(\xi, \tau)\right)-f(\xi, \tau, 0)\right] e^{-(2 \pi k)^{2}(t-\tau)}(1-\xi) \sin 2 \pi k \xi d \xi d \tau \\
& +4 \int_{0}^{t} \int_{0}^{1} f(\xi, \tau, 0) e^{-(2 \pi k)^{2}(t-\tau)}(1-\xi) \sin 2 \pi k \xi d \xi d \tau \\
& -16 \pi k \int_{0}^{t} \int_{0}^{1}\left[f\left(\xi, \tau, u^{(0)}(\xi, \tau)\right)-f(\xi, \tau, 0)\right](t-\tau) e^{-(2 \pi k)^{2}(t-\tau)} \cos 2 \pi k \xi d \xi d \tau \\
& -16 \pi k \int_{0}^{t} \int_{0}^{1}(t-\tau) f(\xi, \tau, 0) e^{-(2 \pi k)^{2}(t-\tau)} \cos 2 \pi k \xi d \xi d \tau .
\end{aligned}
$$

Applying the Cauchy inequality, the Hölder inequality, the Lipschitz condition and the Bessel inequality to the last equation, we obtain

$$
\begin{aligned}
& \left\|u^{(1)}(t)-u^{(0)}(t)\right\|_{\mathbf{B}_{1}} \leq\left(2 \sqrt{T}+\frac{2 \sqrt{3}}{3}+4 \sqrt{2} T\right)\|b(x, t)\|_{L_{2}(D)}\left\|u^{(0)}(t)\right\|_{\mathbf{B}_{1}} \\
& +\left(2 \sqrt{T}+\frac{2 \sqrt{3}}{3}+4 \sqrt{2} T\right)\|f(x, t, 0)\|_{L_{2}(D)}, \\
& K=\left(2 \sqrt{T}+\frac{2 \sqrt{3}}{3}+4 \sqrt{2} T\right)\|b(x, t)\|_{L_{2}(D)}\left\|u^{(0)}(t)\right\|_{\mathbf{B}_{1}} \\
& +\left(2 \sqrt{T}+\frac{2 \sqrt{3}}{3}+4 \sqrt{2} T\right)\|f(x, t, 0)\|_{L_{2}(D)}, \\
& u_{0}^{(2)}(t)-u_{0}^{(1)}(t)=2 \int_{0}^{t} \int_{0}^{1}\left[f\left(\xi, \tau, u^{(1)}(\xi, \tau)\right)-f\left(\xi, \tau, u^{(0)}(\xi, \tau)\right)\right] e^{-\int_{\tau}^{t} p^{(1)}(s) d s} d \xi d \tau \\
& +2 \int_{0}^{t} \int_{0}^{1} f\left(\xi, \tau, u^{(0)}(\xi, \tau)\right)\left[e^{-\int_{\tau}^{t} p^{(1)}(s) d s}-e^{-\int_{\tau}^{t} p^{(0)}(s) d s}\right] d \xi d \tau, \\
& u_{2 k-1}^{(2)}(t)-u_{2 k-1}^{(1)}(t) \\
& =4 \int_{0}^{t} \int_{0}^{1}\left[f\left(\xi, \tau, u^{(1)}(\xi, \tau)\right)-f\left(\xi, \tau, u^{(0)}(\xi, \tau)\right)\right] \\
& \times e^{-(2 \pi k)^{2}(t-\tau)} e^{-\int_{\tau}^{t} p^{(1)}(s) d s} \cos 2 \pi k \xi d \xi d \tau \\
& +4 \int_{0}^{t} \int_{0}^{1} f\left(\xi, \tau, u^{(0)}(\xi, \tau)\right) \cos 2 \pi k \xi e^{-(2 \pi k)^{2}(t-\tau)}\left[e^{-\int_{\tau}^{t} p^{(1)}(s) d s}-e^{-\int_{\tau}^{t} p^{(0)}(s) d s}\right] d \xi d \tau, \\
& u_{2 k}^{(2)}(t)-u_{2 k}^{(1)}(t)=4 \int_{0}^{t} \int_{0}^{1}\left[f\left(\xi, \tau, u^{(1)}(\xi, \tau)\right)-f\left(\xi, \tau, u^{(0)}(\xi, \tau)\right)\right] \\
& \times e^{-(2 \pi k)^{2}(t-\tau)} e^{-\int_{\tau}^{t} p^{(1)}(s) d s}(1-\xi) \sin 2 \pi k \xi d \xi d \tau \\
& +4 \int_{0}^{t} \int_{0}^{1} f\left(\xi, \tau, u^{(0)}(\xi, \tau)\right)(1-\xi) \sin 2 \pi k \xi e^{-(2 \pi k)^{2}(t-\tau)} \\
& \times\left[e^{-\int_{\tau}^{t} p^{(1)}(s) d s}-e^{-\int_{\tau}^{t} p^{(0)}(s) d s}\right] d \xi d \tau \\
& -16 \pi k \int_{0}^{t} \int_{0}^{1}(t-\tau)\left[f\left(\xi, \tau, u^{(1)}(\xi, \tau)\right)-f\left(\xi, \tau, u^{(0)}(\xi, \tau)\right)\right] \\
& \times e^{-(2 \pi k)^{2}(t-\tau)} e^{-\int_{\tau}^{t} p^{(1)}(s) d s} \cos 2 \pi k \xi d \xi d \tau
\end{aligned}
$$




$$
\begin{aligned}
& -16 \pi k \int_{0}^{t} \int_{0}^{1}(t-\tau) f\left(\xi, \tau, u^{(0)}(\xi, \tau)\right) e^{-(2 \pi k)^{2}(t-\tau)} \\
& \times\left[e^{-\int_{\tau}^{t} p^{(1)}(s) d s}-e^{-\int_{\tau}^{t} p^{(0)}(s) d s}\right] \cos 2 \pi k \xi d \xi d \tau
\end{aligned}
$$

Applying the Cauchy inequality, the Hölder inequality, the Lipschitz condition and the Bessel inequality to the last equation, we obtain

$$
\begin{aligned}
&\left\|u^{(2)}(t)-u^{(1)}(t)\right\|_{\mathbf{B}_{1}} \leq\left(2 \sqrt{T}+\frac{2 \sqrt{3}}{3}+4 \sqrt{2} T\right)\|b(x, t)\|_{L_{2}(D)}\left\|u^{(1)}(t)-u^{(0)}(t)\right\|_{\mathbf{B}_{1}} \\
&+\left(2 \sqrt{T}+\frac{2 \sqrt{3}}{3}+4 \sqrt{2} T\right)|T|\|f(x, t, 0)\|_{L_{2}(D)}\left\|p^{(1)}-p^{(0)}\right\|_{\mathbf{B}_{2}}, \\
& p^{(1)}-p^{(0)}=\frac{1}{E(t)} \int_{0}^{1}\left[f\left(\xi, \tau, u^{(1)}\right)-f\left(\xi, \tau, u^{(0)}\right)\right] d \xi .
\end{aligned}
$$

Applying the Cauchy inequality and the Lipschitz condition to the last equation, we obtain

$$
\begin{aligned}
& \left\|p^{(1)}-p^{(0)}\right\|_{\mathbf{B}_{2}} \leq \frac{1}{E(t)}\|b(x, t)\|_{L_{2}(D)}\left\|u^{(1)}(t)-u^{(0)}(t)\right\|_{B_{1}}, \\
& \left\|u^{(2)}(t)-u^{(1)}(t)\right\|_{\mathbf{B}_{1}} \leq\left(2 \sqrt{T}+\frac{2 \sqrt{3}}{3}+4 \sqrt{2} T\right)\|b(x, t)\|_{L_{2}(D)}\left\|u^{(1)}(t)-u^{(0)}(t)\right\|_{\mathbf{B}_{1}} \\
& +\left(2 \sqrt{T}+\frac{2 \sqrt{3}}{3}+4 \sqrt{2} T\right) \frac{T M}{E(t)}\|b(x, t)\|_{L_{2}(D)}\left\|u^{(1)}(t)-u^{(0)}(t)\right\|_{\mathbf{B}_{1}}, \\
& \left\|u^{(2)}(t)-u^{(1)}(t)\right\|_{\mathbf{B}_{1}} \leq\left(2 \sqrt{T}+\frac{2 \sqrt{3}}{3}+4 \sqrt{2} T\right)\left(1+\frac{T M}{E(t)}\right)\|b(x, t)\|_{L_{2}(D)} K, \\
& u_{0}^{(3)}(t)-u_{0}^{(2)}(t)=2 \int_{0}^{t} \int_{0}^{1}\left[f\left(\xi, \tau, u^{(2)}(\xi, \tau)\right)-f\left(\xi, \tau, u^{(1)}(\xi, \tau)\right)\right] e^{-\int_{\tau}^{t} p^{(2)}(s) d s} d \xi d \tau \\
& +2 \int_{0}^{t} \int_{0}^{1} f\left(\xi, \tau, u^{(1)}(\xi, \tau)\right)\left[e^{-\int_{\tau}^{t} p^{(2)}(s) d s}-e^{-\int_{\tau}^{t} p^{(1)}(s) d s}\right] d \xi d \tau, \\
& u_{2 k-1}^{(3)}(t)-u_{2 k-1}^{(2)}(t)=4 \int_{0}^{t} \int_{0}^{1}\left[f\left(\xi, \tau, u^{(2)}(\xi, \tau)\right)-f\left(\xi, \tau, u^{(1)}(\xi, \tau)\right)\right] \\
& \times e^{-(2 \pi k)^{2}(t-\tau)} e^{-\int_{\tau}^{t} p^{(2)}(s) d s} \cos 2 \pi k \xi d \xi d \tau \\
& +4 \int_{0}^{t} \int_{0}^{1} f\left(\xi, \tau, u^{(1)}(\xi, \tau)\right) \cos 2 \pi k \xi e^{-(2 \pi k)^{2}(t-\tau)} \\
& \times\left[e^{-\int_{\tau}^{t} p^{(2)}(s) d s}-e^{-\int_{\tau}^{t} p^{(1)}(s) d s}\right] d \xi d \tau, \\
& u_{2 k}^{(3)}(t)-u_{2 k}^{(2)}(t)=4 \int_{0}^{t} \int_{0}^{1}\left[f\left(\xi, \tau, u^{(2)}(\xi, \tau)\right)-f\left(\xi, \tau, u^{(1)}(\xi, \tau)\right)\right] \\
& \times e^{-(2 \pi k)^{2}(t-\tau)} e^{-\int_{\tau}^{t} p^{(2)}(s) d s}(1-\xi) \sin 2 \pi k \xi d \xi d \tau \\
& +4 \int_{0}^{t} \int_{0}^{1} f\left(\xi, \tau, u^{(1)}(\xi, \tau)\right)(1-\xi) \sin 2 \pi k \xi e^{-(2 \pi k)^{2}(t-\tau)} \\
& \times\left[e^{-\int_{\tau}^{t} p^{(2)}(s) d s}-e^{-\int_{\tau}^{t} p^{(1)}(s) d s}\right] d \xi d \tau \\
& -16 \pi k \int_{0}^{t} \int_{0}^{1}(t-\tau)\left[f\left(\xi, \tau, u^{(2)}(\xi, \tau)\right)-f\left(\xi, \tau, u^{(1)}(\xi, \tau)\right)\right]
\end{aligned}
$$




$$
\begin{aligned}
& \times e^{-(2 \pi k)^{2}(t-\tau)} e^{-\int_{\tau}^{t} p^{(2)}(s) d s} \cos 2 \pi k \xi d \xi d \tau \\
& -16 \pi k \int_{0}^{t} \int_{0}^{1}(t-\tau) f\left(\xi, \tau, u^{(1)}(\xi, \tau)\right) e^{-(2 \pi k)^{2}(t-\tau)} \\
& \times\left[e^{-\int_{\tau}^{t} p^{(2)}(s) d s}-e^{-\int_{\tau}^{t} p^{(1)}(s) d s}\right] \cos 2 \pi k \xi d \xi d \tau .
\end{aligned}
$$

Applying the Cauchy inequality, the Hölder inequality, the Lipschitz condition and the Bessel inequality to the last equation, we obtain

$$
\begin{aligned}
& \left\|u^{(3)}(t)-u^{(2)}(t)\right\|_{\mathbf{B}_{1}} \\
& \leq\left(2 \sqrt{T}+\frac{2 \sqrt{3}}{3}+4 \sqrt{2} T\right)\left(\int_{0}^{t} \int_{0}^{1} b^{2}(\xi, \tau)\left|u^{(2)}(\tau)-u^{(1)}(\tau)\right|^{2} d \xi d \tau\right)^{\frac{1}{2}} \\
& +\left(2 \sqrt{T}+\frac{2 \sqrt{3}}{3}+4 \sqrt{2} T\right) T M\left(\int_{0}^{t} \int_{0}^{1}\left|p^{(2)}(\tau)-p^{(1)}(\tau)\right|^{2} d \xi d \tau\right)^{\frac{1}{2}}, \\
& \left\|u^{(3)}(t)-u^{(2)}(t)\right\|_{\mathbf{B}_{1}} \leq\left(\left(2 \sqrt{T}+\frac{2 \sqrt{3}}{3}+4 \sqrt{2} T\right)\left(1+\frac{T M}{E(t)}\right)\right)^{2} \\
& \times K\left(\int_{0}^{t} \int_{0}^{1} b^{2}(\xi, \tau)\left|u^{(2)}(\tau)-u^{(1)}(\tau)\right|^{2} d \xi d \tau\right)^{\frac{1}{2}}, \\
& \left\|u^{(3)}(t)-u^{(2)}(t)\right\|_{\mathbf{B}_{1}} \leq\left(\left(2 \sqrt{T}+\frac{2 \sqrt{3}}{3}+4 \sqrt{2} T\right)\left(1+\frac{T M}{E(t)}\right)\right)^{2} \\
& \times K\left(\int_{0}^{t} \int_{0}^{1} b^{2}(\xi, \tau)\left(\left(\int_{0}^{t} \int_{0}^{1} b^{2}\left(\xi_{1}, \tau_{1}\right)\right) d \xi_{1} d \tau_{1}\right) d \xi d \tau\right)^{\frac{1}{2}}, \\
& \left\|u^{(3)}(t)-u^{(2)}(t)\right\|_{\mathbf{B}_{1}} \\
& \leq\left(\left(2 \sqrt{T}+\frac{2 \sqrt{3}}{3}+4 \sqrt{2} T\right)\left(1+\frac{T M}{E(t)}\right)\right)^{2} \frac{K}{\sqrt{2}}\left(\left(\int_{0}^{t} \int_{0}^{1} b^{2}(\xi, \tau) d \xi d \tau\right)^{2}\right)^{\frac{1}{2}}, \\
& \left\|u^{(3)}(t)-u^{(2)}(t)\right\|_{\mathbf{B}_{1}} \\
& \leq\left(\left(2 \sqrt{T}+\frac{2 \sqrt{3}}{3}+4 \sqrt{2} T\right)\left(1+\frac{T M}{E(t)}\right)\right)^{2} \frac{K}{\sqrt{2}}\left(\int_{0}^{t} \int_{0}^{1} b^{2}(\xi, \tau) d \xi d \tau\right) .
\end{aligned}
$$

By the same way, we obtain

$$
\begin{aligned}
& \left\|u^{(4)}(t)-u^{(3)}(t)\right\|_{\mathbf{B}_{1}} \\
& \quad \leq\left(\left(2 \sqrt{T}+\frac{2 \sqrt{3}}{3}+4 \sqrt{2} T\right)\left(1+\frac{T M}{E(t)}\right)\right)^{3} \frac{K}{\sqrt{2} \sqrt{3}}\left(\int_{0}^{t} \int_{0}^{1} b^{2}(\xi, \tau) d \xi d \tau\right)^{3} .
\end{aligned}
$$

For $N$, we have

$$
\begin{aligned}
& \left\|p^{(N+1)}-p^{(N)}\right\|_{\mathbf{B}_{2}} \leq\left|\frac{1}{E(t)}\right|\|b(x, t)\|_{L_{2}(D)}\left\|u^{(N+1)}(t)-u^{(N)}(t)\right\|_{\mathbf{B}_{1}}, \\
& \left\|u^{(N+1)}(t)-u^{(N)}(t)\right\|_{\mathbf{B}_{1}} \\
& \leq \frac{K}{\sqrt{N !}}\left(\left(2 \sqrt{T}+\frac{2 \sqrt{3}}{3}+4 \sqrt{2} T\right)\left(1+\frac{T M}{E(t)}\right)\right)^{N}\|b(x, t)\|_{L_{2}(D)}^{N} .
\end{aligned}
$$


Using $\left(A_{1}\right)-\left(A_{3}\right)$ and the comparison test, we deduce from (9) that the series $\sum_{N=0}^{\infty}\left[u^{(N+1)}(t)-u^{(N)}(t)\right]$ is uniformly convergent to an element of $\mathbf{B}_{1}$. However, the general term of the sequence $\left\{u^{(N+1)}(t)\right\}$ may be written as

$$
u^{(N+1)}(t)=u^{(0)}(t)+\sum_{N=0}^{\infty}\left[u^{(N+1)}(t)-u^{(N)}(t)\right]
$$

So the sequence $\left\{u^{(N+1)}(t)\right\}$ is uniformly convergent to an element of $\mathbf{B}_{1}$ because the sum on the right-hand side is the $N$ th partial sum of the aforementioned uniformly convergent series.

It is easy to see that if $u^{(N+1)} \rightarrow u^{(N)}, N \rightarrow \infty$, then $p^{(N+1)} \rightarrow p^{(N)}, N \rightarrow \infty$.

Therefore $u^{(N+1)}(t)$ and $p^{(N+1)}(t)$ converge in $\mathbf{B}_{1}$ and $\mathbf{B}_{2}$, respectively.

Now let us show that there exist $u$ and $p$ such that

$$
\lim _{N \rightarrow \infty} u^{(N+1)}(t)=u(t), \quad \lim _{N \rightarrow \infty} p^{(N+1)}(t)=p(t) .
$$

In the same way, we have

$$
\begin{aligned}
\| u(t) & -u^{(N+1)}(t) \|_{\mathbf{B}_{1}} \\
\leq & \left(2 \sqrt{T}+\frac{2 \sqrt{3}}{3}+4 \sqrt{2} T\right)\|b(x, t)\|_{L_{2}(D)}\left\|u(t)-u^{(N+1)}(t)\right\|_{\mathbf{B}_{1}} \\
& +\left(2 \sqrt{T}+\frac{2 \sqrt{3}}{3}+4 \sqrt{2} T\right)\|b(x, t)\|_{L_{2}(D)}\left\|u^{(N+1)}(\tau)-u^{(N)}(\tau)\right\|_{\mathbf{B}_{1}} \\
& +\left(2 \sqrt{T}+\frac{2 \sqrt{3}}{3}+4 \sqrt{2} T\right)|T|\left\|p(\tau)-p^{(N)}(\tau)\right\|_{\mathbf{B}_{2}}\|f(x, t, u)\|_{L_{2}(D)} \\
\left\|p(t)-p^{(N)}(t)\right\|_{\mathbf{B}_{2}} \leq \frac{1}{E(t)}\left(\int_{0}^{t} \int_{0}^{1} b^{2}(\xi, \tau)\left|u(\tau)-u^{(N+1)}(\tau)\right|^{2} d \xi d \tau\right)^{\frac{1}{2}} & +\frac{1}{E(t)}\left(\int_{0}^{t} \int_{0}^{1} b^{2}(\xi, \tau)\left|u^{(N+1)}(\tau)-u^{(N)}(\tau)\right|^{2} d \xi d \tau\right)^{\frac{1}{2}} .
\end{aligned}
$$

Applying Gronwall's inequality to (10) and using inequalities (9) and (11), we have

$$
\begin{aligned}
\left\|u(t)-u^{(N+1)}(t)\right\|_{\mathbf{B}_{1}} \leq & 2\left[\frac{K}{\sqrt{N !}} D^{2} E^{2}\|b(x, t)\|_{L_{2}(D)}\right]^{2} \\
& \times \exp 2\left(D+D^{2}|T| \frac{M A}{1-B}\right)^{2}\|b(x, t)\|_{L_{2}(D)}^{2} .
\end{aligned}
$$

Here

$$
D=\left(2 \sqrt{T}+\frac{2 \sqrt{3}}{3}+4 \sqrt{2} T\right), \quad E=\left\{\left(2 \sqrt{T}+\frac{2 \sqrt{3}}{3}+4 \sqrt{2} T\right)\left(1+\frac{T M}{|E(t)|}\right)\right\}^{N} .
$$

When $N \rightarrow \infty$, we obtain $u^{(N+1)} \rightarrow u$. Hence $p^{(N+1)} \rightarrow p$.

For the uniqueness, we assume that problem (1)-(4) has two solution pairs $(p, u),(q, v)$. Applying the Cauchy inequality, the Hölder inequality, the Lipschitz condition and the 
Bessel inequality to $|u(t)-v(t)|$ and $|p(t)-q(t)|$, we obtain

$$
\begin{aligned}
\|u(t)-v(t)\|_{\mathbf{B}_{1} \leq} \leq & \left(\|\varphi\|+\left(2 \sqrt{T}+\frac{2 \sqrt{3}}{3}+4 \sqrt{2} T\right) M\right) T\|p(t)-q(t)\|_{\mathbf{B}_{2}} \\
& +\left(2 \sqrt{T}+\frac{2 \sqrt{3}}{3}+4 \sqrt{2} T\right)\left(\int_{0}^{t} \int_{0}^{\pi} b^{2}(\xi, \tau)|u(\tau)-v(\tau)|^{2} d \xi d \tau\right)^{\frac{1}{2}}, \\
\|p(t)-q(t)\|_{\mathbf{B}_{2} \leq} \leq & \frac{1}{E(t)}\left(\int_{0}^{t} \int_{0}^{1} b^{2}(\xi, \tau)|u(\tau)-v(\tau)|^{2} d \xi d \tau\right)^{\frac{1}{2}}, \\
\|u(t)-v(t)\|_{\mathbf{B}_{1} \leq} \leq & {\left[\left(\|\varphi\|+\left(2 \sqrt{T}+\frac{2 \sqrt{3}}{3}+4 \sqrt{2} T\right) M\right) \frac{T M}{E(t)}\right.} \\
& \left.+\left(2 \sqrt{T}+\frac{2 \sqrt{3}}{3}+4 \sqrt{2} T\right)\right]\left(\int_{0}^{t} \int_{0}^{1} b^{2}(\xi, \tau)|u(\tau)-v(\tau)|^{2} d \xi d \tau\right)^{\frac{1}{2}} .
\end{aligned}
$$

Applying the Gronwall inequality to (13), we have $u(t)=v(t)$. Hence $p(t)=q(t)$.

The theorem is proved.

\section{Continuous dependence of $(p, u)$ upon the data}

Theorem 4 Under assumptions $\left(\mathrm{A}_{1}\right)-\left(\mathrm{A}_{3}\right)$, the solution $(p, u)$ of problem $(1)-(4)$ depends continuously upon the data $\varphi, E$.

Proof Let $\Phi=\{\varphi, E, f\}$ and $\bar{\Phi}=\{\bar{\varphi}, \bar{E}, f\}$ be two sets of the data, which satisfy the assumptions $\left(\mathrm{A}_{1}\right)-\left(\mathrm{A}_{3}\right)$. Suppose that there exist positive constants $M_{i}, i=0,1,2$, such that

$$
\begin{aligned}
& 0<M_{0} \leq|E|, \quad 0<M_{0} \leq|\bar{E}|, \quad\|E\|_{C^{1}[0, T]} \leq M_{1}, \quad\|\bar{E}\|_{C^{1}[0, T]} \leq M_{1}, \\
& \|\varphi\|_{C^{3}[0,1]} \leq M_{2}, \quad\|\bar{\varphi}\|_{C^{3}[0,1]} \leq M_{2} .
\end{aligned}
$$

Let us denote $\|\Phi\|=\left(\|E\|_{C^{1}[0, T]}+\|\varphi\|_{C^{3}[0,1]}+\|f\|_{C^{3,0}(\bar{D})}\right)$. Let $(p, u)$ and $(\bar{p}, \bar{u})$ be solutions of inverse problem (1)-(4) corresponding to the data $\Phi=\{\varphi, E, f\}$ and $\bar{\Phi}=\{\bar{\varphi}, \bar{E}, f\}$, respectively. According to (5), we have

$$
\begin{aligned}
u(t)-\bar{u}(t)= & 2\left(\varphi_{0}-\overline{\varphi_{0}}\right) e^{-\int_{0}^{t} \bar{p}(s) d s}+2 \varphi_{0}\left(e^{-\int_{0}^{t} p(s) d s}-e^{-\int_{0}^{t} \bar{p}(s) d s}\right) \\
& +2 \int_{0}^{t} \int_{0}^{1}[f(\xi, \tau, u(\xi, \tau))-f(\xi, \tau, \bar{u}(\xi, \tau))] e^{-\int_{\tau}^{t} \bar{p}(s) d s} d \xi d \tau \\
& +2 \int_{0}^{t} \int_{0}^{1} f(\xi, \tau, u(\xi, \tau))\left[e^{-\int_{\tau}^{t} p(s) d s}-e^{-\int_{\tau}^{t} \bar{p}(s) d s}\right] d \xi d \tau \\
& +4 \sum_{k=1}^{\infty} \xi \cos 2 \pi k \xi\left(\varphi_{2 k-1}-\overline{\varphi_{2 k-1}}\right) e^{-(2 \pi k)^{2} t}\left[e^{-\int_{\tau}^{t} p(s) d s}-e^{-\int_{\tau}^{t} \bar{p}(s) d s}\right] \\
& +4 \sum_{k=1}^{\infty} \xi \cos 2 \pi k \xi \varphi_{2 k} e^{-(2 \pi k)^{2} t} e^{-\int_{\tau}^{t} \bar{p}(s) d s} \\
& +4 \sum_{k=1}^{\infty} \sin 2 \pi k \xi\left(\varphi_{2 k}-\overline{\varphi_{2 k}}\right) e^{-(2 \pi k)^{2} t}\left[e^{-\int_{\tau}^{t} p(s) d s}-e^{-\int_{\tau}^{t} \bar{p}(s) d s}\right] \\
& +4 \sum_{k=1}^{\infty} \sin 2 \pi k \xi \varphi_{2 k-1} e^{-(2 k)^{2} t} e^{-\int_{\tau}^{t} \bar{p}(s) d s}
\end{aligned}
$$




$$
\begin{aligned}
& -16 \pi \sum_{k=1}^{\infty} k t \sin 2 \pi k \xi\left(\varphi_{2 k-1}-\overline{\varphi_{2 k-1}}\right) e^{-(2 \pi k)^{2} t}\left[e^{-\int_{\tau}^{t} p(s) d s}-e^{-\int_{\tau}^{t} \bar{p}(s) d s}\right] \\
& -16 \pi \sum_{k=1}^{\infty} k t \sin 2 \pi k \xi \varphi_{2 k} e^{-(2 \pi k)^{2} t} e^{-\int_{\tau}^{t} \bar{p}(s) d s} \\
& +4 \sum_{k=1}^{\infty} \int_{0}^{t} \int_{0}^{1}[f(\xi, \tau, u(\xi, \tau))-f(\xi, \tau, \bar{u}(\xi, \tau))](1-\xi) \sin 2 \pi k \xi \\
& \times e^{-(2 \pi k)^{2}(t-\tau)-\int_{\tau}^{t} \bar{p}(s) d s} d \xi d \tau \\
& +4 \sum_{k=1}^{\infty} \int_{0}^{t} \int_{0}^{1} f(\xi, \tau, u(\xi, \tau))(1-\xi) \sin 2 \pi k \xi e^{-(2 \pi k)^{2}(t-\tau)} \\
& \times\left[e^{-\int_{\tau}^{t} p(s) d s}-e^{-\int_{\tau}^{t} \bar{p}(s) d s}\right] d \xi d \tau \\
& +4 \sum_{k=1}^{\infty} \int_{0}^{t} \int_{0}^{1}[f(\xi, \tau, u(\xi, \tau))-f(\xi, \tau, \bar{u}(\xi, \tau))](1-\xi) \sin 2 \pi k \xi \\
& \times e^{-(2 \pi k)^{2}(t-\tau)-\int_{\tau}^{t} \bar{p}(s) d s} d \xi d \tau \\
& +4 \sum_{k=1}^{\infty} \int_{0}^{t} \int_{0}^{1} f(\xi, \tau, u(\xi, \tau))(1-\xi) \sin 2 \pi k \xi e^{-(2 \pi k)^{2}(t-\tau)} \\
& \times\left[e^{-\int_{\tau}^{t} p(s) d s}-e^{-\int_{\tau}^{t} \bar{p}(s) d s}\right] d \xi d \tau \\
& -16 \pi \sum_{k=1}^{\infty} k \int_{0}^{t} \int_{0}^{1}[f(\xi, \tau, u(\xi, \tau))-f(\xi, \tau, \bar{u}(\xi, \tau))](t-\tau) \cos 2 \pi k \xi \\
& \times e^{-(2 \pi k)^{2}(t-\tau)-\int_{\tau}^{t} \bar{p}(s) d s} d \xi d \tau \\
& -16 \pi \sum_{k=1}^{\infty} k \int_{0}^{t} \int_{0}^{1} f(\xi, \tau, u(\xi, \tau))(t-\tau) \cos 2 \pi k \xi e^{-(2 \pi k)^{2}(t-\tau)} \\
& \times\left[e^{-\int_{\tau}^{t} p(s) d s}-e^{-\int_{\tau}^{t} \bar{p}(s) d s}\right] d \xi d \tau, \\
& |u(t)-\bar{u}(t)| \leq\left(\left(2 \sqrt{T}+\frac{2 \sqrt{3}}{3}+4 \sqrt{2} T\right)+\frac{2 \pi^{2} \sqrt{6}}{3} \sum_{k=0}^{\infty}\left|\varphi_{2 k}^{\prime \prime \prime}\right|+\sum_{k=0}^{\infty}\left(\left|\varphi_{2 k}\right|+\left|\varphi_{2 k-1}\right|\right)\right) \\
& \times\|p-\bar{p}\|_{B_{2}}+\left(1+\frac{2 \sqrt{6}}{3} T\right)\|\varphi-\bar{\varphi}\|_{C^{3}[0,1]} \\
& +\left(2 \sqrt{T}+\frac{2 \sqrt{3}}{3}+4 \sqrt{2} T\right)\left(\int_{0}^{t} \int_{0}^{1} b^{2}(\xi, \tau)|u(\tau)-\bar{u}(\tau)|^{2} d \xi d \tau\right)^{\frac{1}{2}} .
\end{aligned}
$$

Now let us estimate the difference $p-\bar{p}$.

$$
\begin{aligned}
p(t)-\bar{p}(t)= & \left(\frac{-E^{\prime}(t)}{E(t)}+\frac{\overline{E^{\prime}(t)}}{\overline{E(t)}}\right)+\frac{1}{E(t)} \int_{0}^{1} f(\xi, \tau, u) d \xi-\frac{1}{\bar{E}(t)} \int_{0}^{1} f(\xi, \tau, \bar{u}) d \xi \\
p(t)-\bar{p}(t)= & \left(\frac{-E^{\prime}(t)}{E(t)}+\frac{\overline{E^{\prime}(t)}}{\overline{E(t)}}\right)+\frac{1}{E(t)} \int_{0}^{1}[f(\xi, \tau, u)-f(\xi, \tau, \bar{u})] d \xi \\
& +\left(\frac{1}{E(t)}-\frac{1}{\bar{E}(t)}\right) \int_{0}^{1} f(\xi, \tau, \bar{u}) d \xi
\end{aligned}
$$


Applying the Cauchy equation, we have

$$
\|p(t)-\bar{p}(t)\|_{\mathbf{B}_{2}} \leq M_{3}\|E-\bar{E}\|_{C^{1}[0, T]}+M_{4}\left(\int_{0}^{t} \int_{0}^{\pi} b^{2}(\xi, \tau)|u(\tau)-\bar{u}(\tau)|^{2} d \xi d \tau\right)^{\frac{1}{2}}
$$

where $M_{k}, k=3,4$, are constants that are determined by $M_{0}, M_{1}$ and $M_{2}$. Finally, we obtain

$$
\|p(t)-\bar{p}(t)\|_{\mathbf{B}_{2}} \leq M_{5}\left(\|E-\bar{E}\|_{C^{1}[0, T]}+\left(\int_{0}^{t} \int_{0}^{\pi} b^{2}(\xi, \tau)|u(\tau)-\bar{u}(\tau)|^{2} d \xi d \tau\right)^{\frac{1}{2}}\right) .
$$

If we take this estimation in (14), we get

$$
\begin{aligned}
& |u(t)-\bar{u}(t)| \leq M_{6}\|\Phi-\bar{\Phi}\|+M_{7}\left(\int_{0}^{t} \int_{0}^{1} b^{2}(\xi, \tau)|u(\tau)-\bar{u}(\tau)|^{2} d \xi d \tau\right)^{\frac{1}{2}}, \\
& |u(t)-\bar{u}(t)| \leq M_{6}\|\Phi-\bar{\Phi}\|+M_{7}\left(\int_{0}^{t} \int_{0}^{1} b^{2}(\xi, \tau)|u(\tau)-\bar{u}(\tau)|^{2} d \xi d \tau\right)^{\frac{1}{2}}, \\
& |u(t)-\bar{u}(t)|^{2} \leq 2 M_{6}^{2}\|\Phi-\bar{\Phi}\|^{2}+2 M_{7}^{2}\left(\int_{0}^{t} \int_{0}^{\pi} b^{2}(\xi, \tau)|u(\tau)-\bar{u}(\tau)|^{2} d \xi d \tau\right) .
\end{aligned}
$$

Applying the Gronwall inequality, we obtain

$$
|u(t)-\bar{u}(t)|^{2} \leq 2 M_{6}^{2}\|\Phi-\bar{\Phi}\|^{2} \exp 2 M_{7}^{2}\left(\int_{0}^{t} \int_{0}^{1} b^{2}(\xi, \tau) d \xi d \tau\right) .
$$

Taking the maximum of the inequality, we have

$$
\|u(t)-\bar{u}(t)\|_{\mathbf{B}_{1}}^{2} \leq 2 M_{6}^{2}\|\Phi-\bar{\Phi}\|^{2} \exp 2 M_{7}^{2}\left(\int_{0}^{t} \int_{0}^{1} b^{2}(\xi, \tau) d \xi d \tau\right) .
$$

If $\Phi \rightarrow \bar{\Phi}$, then $u \rightarrow \bar{u}$. Hence $p \rightarrow \bar{p}$.

\section{Numerical procedure for nonlinear problem (1)-(4)}

We construct an iteration algorithm for the linearization of problem (1)-(4) as follows.

$$
\begin{aligned}
& \frac{\partial u^{(n)}}{\partial t}=\frac{\partial^{2} u^{(n)}}{\partial x^{2}}-p(t) u^{(n)}+f\left(x, t, u^{(n-1)}\right), \quad(x, t) \in D, \\
& u^{(n)}(0, t)=0, \quad t \in[0, T], \\
& u_{x}^{(n)}(0, t)=u_{x}^{(n)}(1, t), \quad t \in[0, T], \\
& u^{(n)}(x, 0)=\varphi(x), \quad x \in[0,1] .
\end{aligned}
$$

Let $u^{(n)}(x, t)=v(x, t)$ and $f\left(x, t, u^{(n-1)}\right)=\tilde{f}(x, t)$. Then problem (15)-(18) can be written as a linear problem:

$$
\begin{aligned}
& \frac{\partial v}{\partial t}=\frac{\partial^{2} v}{\partial x^{2}}-p(t) v(x, t)+\tilde{f}(x, t), \quad(x, t) \in D, \\
& v(0, t)=0, \quad t \in[0, T]
\end{aligned}
$$




$$
\begin{aligned}
& v_{x}(0, t)=v_{x}(1, t), \quad t \in[0, T], \\
& v(x, 0)=\varphi(x), \quad x \in[0,1] .
\end{aligned}
$$

We use the finite difference method to solve (19)-(22) with a predictor-corrector type approach which was explained in [10].

We subdivide the intervals $[0,1]$ and $[0, T]$ into subintervals $N_{x}$ and $N_{t}$ of equal lengths $h=\frac{1}{N_{x}}$ and $\tau=\frac{T}{N_{t}}$, respectively. Then we add two lines $x=0$ and $x=\left(N_{x}+1\right) h$ to generate the fictitious points needed for dealing with the boundary conditions. We choose the implicit scheme that is absolutely stable and has a second-order accuracy in $h$ and a first-order accuracy in $\tau$ [11]. The implicit scheme for (1)-(4) is as follows:

$$
\begin{aligned}
& \frac{1}{\tau}\left(v_{i}^{j+1}-v_{i}^{j}\right)=\frac{1}{h^{2}}\left(v_{i-1}^{j+1}-2 v_{i}^{j+1}+v_{i+1}^{j+1}\right) \\
& \quad-p^{j+1} v_{i}^{j+1}+\widetilde{f}_{i}^{j+1}, \\
& v_{i}^{0}=\phi_{i}, \\
& v_{0}^{j}=0, \\
& v_{N_{x+1}}^{j}=v_{1}^{j}+v_{N_{x}}^{j},
\end{aligned}
$$

where $1 \leq i \leq N_{x}$ and $1 \leq j \leq N_{t}$ are the indices for the spatial and time steps, respectively, $v_{i}^{j}=v\left(x_{i}, t_{j}\right), \phi_{i}=\varphi\left(x_{i}\right), \tilde{f}_{i}^{j}=\tilde{f}\left(x_{i}, t_{j}\right), x_{i}=i h, t_{j}=j \tau$. At the level $t=0$, adjustment should be made according to the initial condition and the compatibility requirements.

The system of equations (17)-(19) can be solved by the Gauss elimination method, and $v_{i}^{j+1}$ is determined.

\section{Numerical example}

Example 1 Consider inverse problem (1)-(4) with

$$
\begin{aligned}
& f(x, t, u)=-4 \pi \sin (2 \pi x) \exp (-t)-x(2 \pi)^{2} \cos (2 \pi x) \exp (-t)+\exp (3 t) u, \\
& \varphi(x)=x(1-\cos (2 \pi x)), \quad E(t)=\frac{1}{2} \exp (-t), \quad x \in[0,1], t \in[0, T] .
\end{aligned}
$$

It is easy to check that the analytical solution of this problem is

$$
\{p(t), u(x, t)\}=\{1+\exp (3 t), x(1-\cos (2 \pi x)) \exp (-t)\} .
$$

Let us apply the scheme which was explained in the previous section to the step sizes $h=0.05, \tau=0.05$.

In the case when $T=1$, the comparisons between the analytical solution (23) and the numerical finite difference solution are shown in Figures 1 and 2.

It is clear from these results that this method has been shown to produce stable and reasonably accurate results for these examples. 


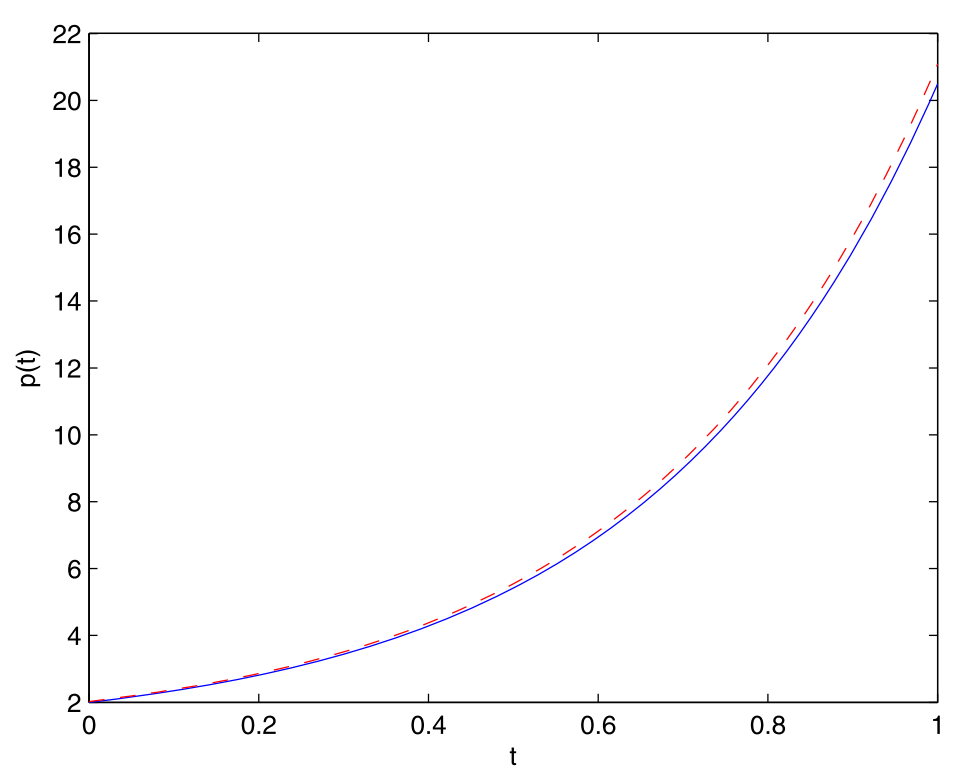

Figure 1 The analytical and numerical solutions of $p(t)$ when $T=1$. The analytical solution is shown with dashed line

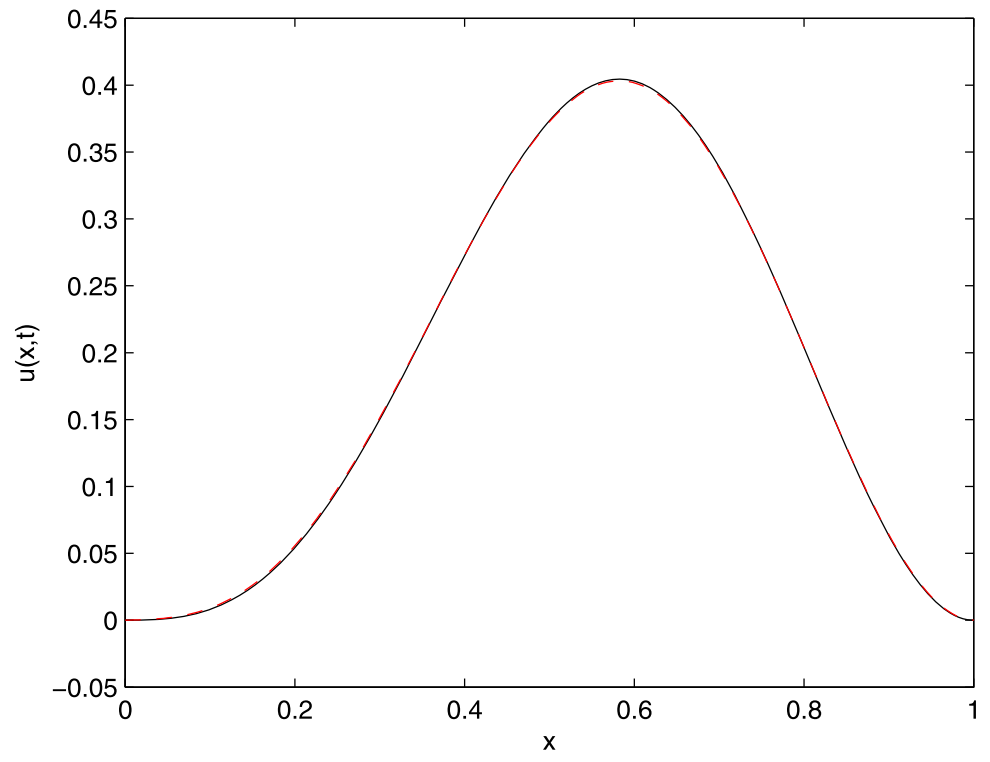

Figure 2 The analytical and numerical solutions of $u(x, t)$ at the $T=1$. The analytical solution is shown with dashed line.

\section{Authors' contributions}

FK conceived the study, participated in its design and coordination and prepared computing section. IB participated in the sequence alignment and achieved the estimation. All authors read and approved the final manuscript.

\section{Author details}

'Department of Management Information Systems, Kadir Has University, Istanbul, 34083, Turkey. ${ }^{2}$ Department of Mathematics, Kocaeli University, Kocaeli, 41380, Turkey. 


\section{References}

1. Cannon, J, Lin, Y: Determination of parameter $p(t)$ in Holder classes for some semilinear parabolic equations. Inverse Probl. 4, 595-606 (1988)

2. Pourgholia, R, Rostamiana, M, Emamjome, M: A numerical method for solving a nonlinear inverse parabolic problem. Inverse Probl. Sci. Eng. 18, 1151-1164 (2010)

3. Gatti, S: An existence result for an inverse problem for a quasilinear parabolic equation. Inverse Probl. 14, 53-65 (1998)

4. Namazov, G: Definition of the unknown coefficient of a parabolic equation with nonlocal boundary and complementary conditions. Trans. Acad. Sci. Azerb. Ser. Phys.-Tech. Math. Sci. 19, 113-117 (1999)

5. Ismailov, M, Kanca, F: An inverse coefficient problem for a parabolic equation in the case of nonlocal boundary and overdetermination conditions. Math. Methods Appl. Sci. 34, 692-702 (2011)

6. Nakhushev, AM: Equations of Mathematical Biology. Moscow (1995)

7. Cannon, J: The solution of the heat equation subject to specification of energy. Q. Appl. Math. 21, 155-160 (1963)

8. Ionkin, N: Solution of a boundary-value problem in heat conduction with a nonclassical boundary condition. Differ. Equ. 13, 204-211 (1977)

9. Kanca, F, Ismailov, M: Inverse problem of finding the time-dependent coefficient of heat equation from integral overdetermination condition data. Inverse Probl. Sci. Eng. 20, 463-476 (2012)

10. Cannon, J, Lin, Y, Wang, S: Determination of source parameter in a parabolic equations. Meccanica 27, 85-94 (1992)

11. Samarskii, AA: The Theory of Difference Schemes. Dekker, New York (2001)

10.1186/1029-242X-2014-76

Cite this article as: Kanca and Baglan: An inverse problem for a quasilinear parabolic equation with nonlocal boundary and overdetermination conditions. Journal of Inequalities and Applications 2014, 2014:76

\section{Submit your manuscript to a SpringerOpen ${ }^{\circ}$ journal and benefit from:}

- Convenient online submission

- Rigorous peer review

- Immediate publication on acceptance

- Open access: articles freely available online

- High visibility within the field

- Retaining the copyright to your article 